\title{
Seasonal sediment dynamics shape temperate bedrock reef communities
}

\author{
Jared D. Figurski ${ }^{1,5, *}$, Jan Freiwald ${ }^{2}$, Steve I. Lonhart ${ }^{3}$, Curt D. Storlazzi ${ }^{4}$ \\ ${ }^{1}$ Ecology and Evolutionary Biology, University of California at Santa Cruz, 100 Shaffer Road, Santa Cruz, CA 95060, USA \\ ${ }^{2}$ Institute of Marine Sciences, University of California at Santa Cruz, 100 Shaffer Road, Santa Cruz, CA 95060, USA \\ ${ }^{3}$ National Oceanic and Atmospheric Administration, Monterey Bay National Marine Sanctuary, 110 Shaffer Road, \\ Santa Cruz, CA 95060, USA
}

${ }^{4}$ US Geological Survey, Pacific Coastal and Marine Science Center, 400 Natural Bridges Drive, Santa Cruz, CA 95060, USA

${ }^{5}$ Present address: Monterey Bay Aquarium Research Institute, 7700 Sandholdt Road, Moss Landing, CA 95039, USA

\begin{abstract}
Mobilized seafloor sediment can impact benthic reef communities through burial, scour, and turbidity. These processes are ubiquitous in coastal oceans and, through their influence on the survival, fitness, and interactions of species, can alter the structure and function of benthic communities. In northern Monterey Bay, California, USA, as much as $30 \%$ of the seafloor is buried or exposed seasonally, making this an ideal location to test how subtidal temperate rocky reef communities vary in the presence and absence of chronic sediment-based disturbances. Designated dynamic plots were naturally inundated by sediment in summer (50 to $100 \%$ cover) and swept clean in winter, whereas designated stable plots remained free of sediment during our study. Multivariate analyses indicated significant differences in the structure of sessile and mobile communities between dynamic and stable reef habitats. For sessile species, community structure in disturbed plots was less variable in space and time than in stable plots due to the maintenance of an early successional state. In contrast, community structure of mobile species varied more in disturbed plots than in stable plots, reflecting how mobile species distribute in response to sediment dynamics. Some species were found only in these disturbed areas, suggesting that the spatial mosaic of disturbance could increase regional diversity. We discuss how the relative ability of species to tolerate disturbance at different life history stages and their ability to colonize habitat translate into community-level differences among habitats, and how this response varies between mobile and sessile communities.
\end{abstract}

KEY WORDS: Benthic $\cdot$ Diversity $\cdot$ Intermediate disturbance hypothesis $\cdot$ Mobile invertebrates · Physical disturbance $\cdot$ Recruitment $\cdot$ Sessile $\cdot$ Seafloor mapping $\cdot$ Kelp forest ecology

\section{INTRODUCTION}

Variation in the intensity, frequency, duration, and spatial extent of physical disturbance has important consequences on the structure (e.g. composition and diversity) and function (e.g. productivity and resilience) of ecosystems (Menge \& Sutherland 1987, Hall 1994, Harris 2014). Small-scale disturbances such as tree falls can remove individuals, thereby altering

*Corresponding author: jared@mbari.org the web of species interactions (i.e. competition, predation, herbivory and facilitation), whereas largescale disturbances such as mudslides, wildfires, and flooding can alter the structure of habitats and reset ecological succession or drive communities to alternate stable states (Thistle 1981, Sousa 2001, Thistle 2003). Although extreme disturbances (i.e. intense and expansive) are important, these events are typically infrequent (Connell 1978, Sousa 1984). In con-

() The authors 2016. Open Access under Creative Commons by Attribution Licence. Use, distribution and reproduction are unrestricted. Authors and original publication must be credited. 
trast, weak disturbances occur more frequently and are consequently thought to be important in structuring biological communities by reducing the abundance of competitive dominant species and favoring species that are early colonizers or tolerant of disturbance (Wu \& Loucks 1995, Svensson et al. 2009). Connell (1978) proposed the intermediate disturbance hypothesis (IDH) to explain why the highest levels of species diversity are often found at intermediate levels of physical disturbance. In his examples, periodic tree falls in tropical forests and episodic storm damage to coral reefs are disturbances that reduce the abundance of competitive dominant species and allow co-existence. Since its proposal, many studies have supported the IDH across a wide variety of ecosystems, and authors have proposed additional mechanisms to explain how intermediate levels of disturbance promote the highest species diversity (Roxburgh et al. 2004). Critics agree that disturbance alters patterns of diversity but highlight empirical and theoretical shortcomings of the IDH (Fox 2013). The continuing debate over the role of disturbance in maintaining species diversity emphasizes the need for further field studies to understand the sources and consequences of disturbance on community structure and functioning.

Few ecosystems are as heavily influenced by physical disturbance as coastal oceans, where powerful ocean waves transfer energy from distant storms directly to seafloor habitats. The forces experienced by benthic organisms on a daily basis from ocean waves rival the strength of hurricane-force winds (Denny \& Gaylord 2002) and are destructive both directly to organisms through battering and dislodgement, and indirectly through the modification of their habitat (Jones et al. 2011). Specialized physiology and behavior allow organisms to withstand strong drag forces at the seabed (Denny \& Gaylord 2002). For example, sessile organisms withstand wave forces by being firmly affixed to the substrate, hydrodynamic, and/or flexible (Denny 1987), whereas mobile benthic organisms can seek refuge by moving to deeper water or to protective seabed structures (Menge \& Sutherland 1987). Mobilization of unconsolidated seafloor sediment by waves can also disturb benthic communities via burial, scour, and turbidity. Margins of reefs, as a result, are dynamic environments where sediment can scour and/or bury vast areas of rocky reef substrate, reduce or eliminate competitive dominant species, create space for recruitment, and potentially reset ecological succession (Littler et al. 1983, McQuaid \& Dower 1990, Airoldi 2003).
Bathymetry generally defines the area of reef that is vulnerable to the influence of sediment dynamics. Where reefs are low in profile, the area of potential burial can be extensive. Along central California, for example, up to $30 \%$ of bedrock reefs are buried or exhumed in a single year (Storlazzi et al. 2011). Vast aprons of shifting sediment sweep across rocky reefs, burying the lowest and scouring the highest parts of the reef. Burial and subsequent exhumation of rocky reefs happen episodically in response to wave events and regularly on a seasonal basis (Storlazzi et al. 2011). This phenomenon is universal in coastal marine environments, but varies spatially in extent and severity depending on bathymetry, sediment, and wave exposure (Field 2005, Ryan et al. 2007, Harris \& Hughes 2012). Historically, it has been difficult to quantify burial at meaningful spatial and temporal scales, but recent advancements in acoustic seafloor mapping technologies allow for repetitive, high-resolution, geo-located surveys of bathymetry and substrate composition. Quantification of seafloor dynamics over vast areas is now possible, allowing, for the first time, a detailed understanding of the timescales of burial and exhumation and how these processes shape benthic marine communities.

In this study, we evaluated how seafloor sediment dynamics influence the species composition and community structure of benthic communities, and how those responses differ between communities of mobile and sessile species. We used inter-annual swath acoustic surveys of the seafloor to identify temperate reef habitats that either experienced seasonal burial and exhumation (dynamic) or did not (stable), then compared the structure and dynamics of biological communities that we quantified with seasonal surveys of benthic organisms using in situ observations made via SCUBA. The results of this study are important for documenting the linkage between seafloor dynamics and disturbance ecology (Harris 2014). Understanding how episodic natural stressors affect benthic communities is becoming increasingly important, particularly in the context of human- and climate-based stressors that are increasing in coastal ecosystems.

\section{MATERIALS AND METHODS}

\section{General approach}

We compared subtidal temperate rocky reef communities in perennially stable habitats with those disturbed by seasonal burial and exhumation. We used 
repetitive SCUBA surveys to quantify species abundance and cover, to describe the composition and structure of benthic communities at 2 locations in northern Monterey Bay, California, USA, in December 2009, April 2010, and August 2010. High-resolution, swath acoustic seafloor surveys were completed at the same time to document the burial and exhumation dynamics on the reefs. Habitat classifications from the acoustic surveys were ground-truthed with in situ measurements by SCUBA divers.

\section{System description}

The southward-facing coastline of northern Monterey Bay offers moderate protection from the prevailing northwest swell in autumn and spring, but wave heights can exceed $6 \mathrm{~m}$ during winter storms (Wingfield \& Storlazzi 2007). Associated seabed forces and currents transport fine and coarse sediment across and along shore (Storlazzi \& Jaffe 2002). The consolidated rocky reef in this area is composed of low-lying (typically $<1 \mathrm{~m}$ high) sedimentary Santa Cruz Mudstone and is prone to burial; the unconsolidated seafloor sediment on the inner continental shelf (depths $<30 \mathrm{~m}$ ) is predominantly sand, with lesser amounts of silts and clays (Anima et al. 2002, Storlazzi et al. 2011). Most of the benthic species found at our study sites are typical for giant kelp Macrocystis pyrifera forests and rocky reefs in central California, even though the study plots were located somewhat deeper $(\sim 2 \mathrm{~m})$ than the offshore boundary of the kelp forests. In the northern region of Monterey Bay, the lower depth limits of many algae are reduced due to light limitation from enhanced phytoplankton productivity (Foster \& Schiel 1985) and from the suspension of fine sediments. In the vicinity of this study, the giant kelp forests typically extend no deeper than $12 \mathrm{~m}$, whereas south of the bay the same kelp regularly occurs to depths in excess of $25 \mathrm{~m}$. The benthic communities are less speciose than their southern counterparts and are dominated by invertebrates such as tunicates, sponges, polychaete worms, and boring clams rather than by macrophytes (Schiel \& Foster 2015).

\section{Identifying study plots and transects}

In this study, we used repetitive high-resolution, swath interferometric side-scan sonar surveys to identify 2 sites - Terrace Point (TP; $36.948006^{\circ} \mathrm{N}$, $122.065940^{\circ} \mathrm{W} ; 14 \mathrm{~m}$ depth) and Lighthouse Point $\left(\mathrm{LP} ; 36.950301^{\circ} \mathrm{N}, 122.026596^{\circ} \mathrm{W} ; 13 \mathrm{~m}\right.$ depth) — that demonstrated burial and exhumation dynamics during the 2 yr prior to this study (Storlazzi et al. 2011). At each site, we chose a single $150 \times 10 \mathrm{~m}$ plot that alternated annually between rock and sediment (hereafter referred to as 'dynamic' plots). For comparison, a single plot at each site with similar physical structure (i.e. depth, bathymetry, and rugosity) but in unchanging reef habitat (i.e. lacking patterns of burial and exhumation) was selected (hereafter referred to as 'stable' plots) adjacent to the dynamic plots (less than $150 \mathrm{~m}$ away) to minimize spatially confounding factors such as variation of wave exposure or recruitment (Fig. 1). Prior to the study, we selected the starting positions and bearings for two $30 \mathrm{~m}$ transects in each of the 4 plots using the swath acoustic seafloor surveys to ensure unbiased placement of the transects by the divers. To maximize independence of these transects, the starting points were separated by $10 \mathrm{~m}$ and transects extended in opposite directions.

Measurements of the biological community and in situ characterizations of the substrate were repeated for the same fixed transects in each sampling period (i.e. winter 2009, spring 2010, and summer 2010). Within a sampling period, all transects were typically surveyed within $3 \mathrm{~d}$.

\section{Sampling sessile benthic communities and substrate}

To quantify the percent cover of sessile, rock-associated organisms, we used a uniform point contact (UPC) protocol developed by the Partnership for Interdisciplinary Studies of Coastal Oceans (PISCO) for monitoring kelp forests in California (Hamilton et al. 2010). Details for these sampling protocols are described at http://www.piscoweb.org/research/scienceby-discipline/ecosystem-monitoring/kelp-forestmonitoring/subtidal-sampling-protoco. Along each of two $30 \mathrm{~m}$ transects per plot, a diver sampled at $0.5 \mathrm{~m}$ intervals ( $\mathrm{n}=60$ points), identifying all organisms lying below each point to the lowest taxonomic level possible in the field. We also noted the type of substrate at each point: bedrock ( $>100 \mathrm{~cm}$ diameter), boulder (10 to $99 \mathrm{~cm}$ diameter), cobble $(<10 \mathrm{~cm}$ diameter), or sand and finer-grained sediment. Vertical relief of the reef was also measured as the maximum vertical distance found in a $0.5 \times 1.0 \mathrm{~m}$ non-overlapping rectangle centered on each point along the transect. In situ measurements of the substrate were compared 


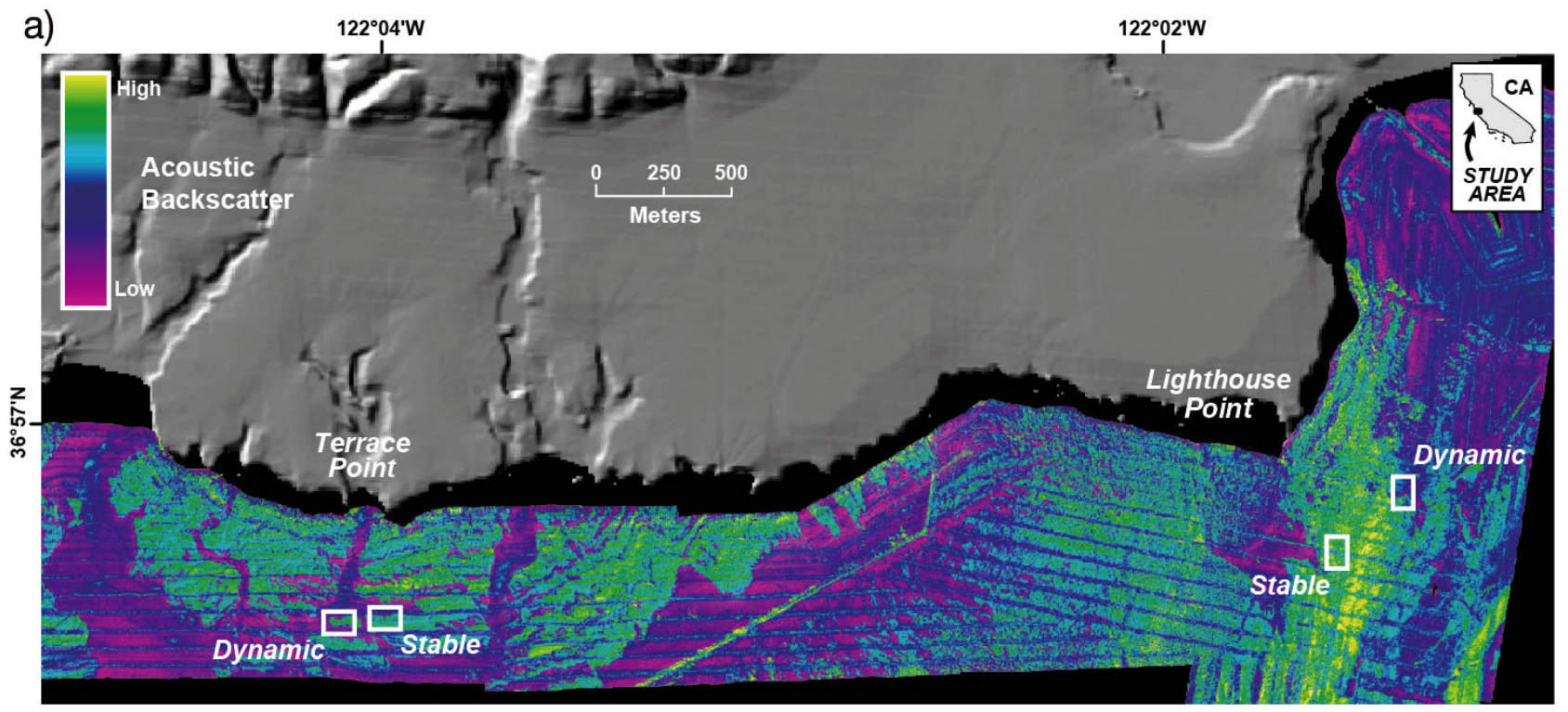

b)

Terrace Point - Dynamic

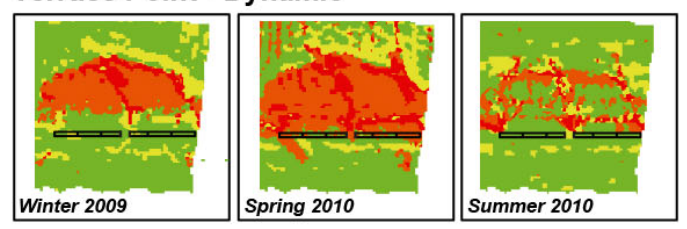

Terrace Point - Stable
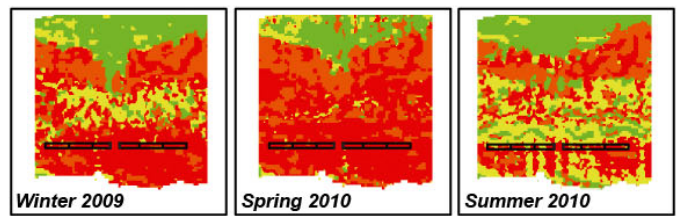

Lighthouse Point - Dynamic

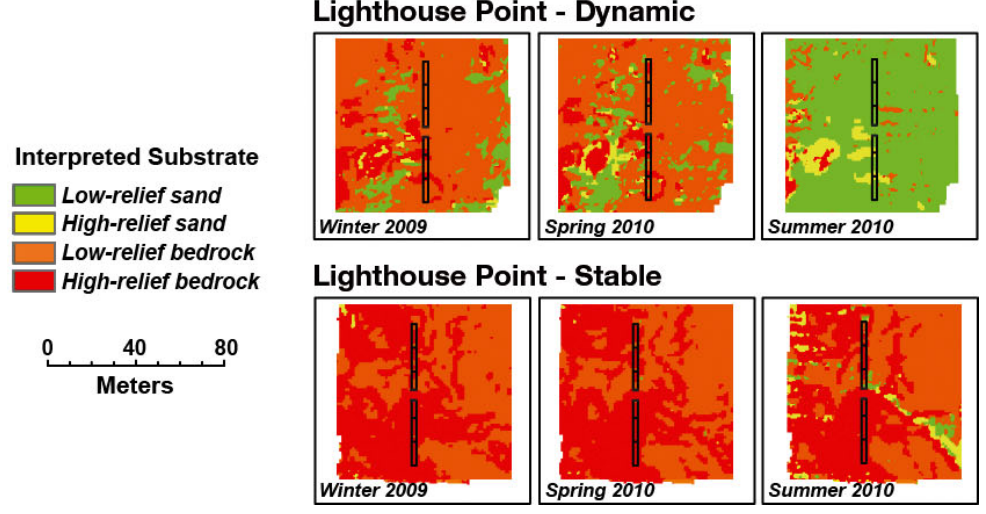

Fig. 1. High-resolution, swath acoustic seafloor map of northern Monterey Bay, California, USA, showing the location, bathymetry, seafloor dynamics, and sampling design of this study. (a) Acoustic surveys reveal a heterogeneous seafloor composed of bedrock (yellows and greens) and sediment (magenta). Stable and dynamic study plots at Terrace Point and Lighthouse Point, selected based on the dynamics observed between 2005 and 2006, are shown as white rectangles. (b) Change in plots over the course of this study with respect to substrate (sediment or bedrock) and relief (high or low). The black lines show where substrate and biological communities were sampled in situ using SCUBA

against concurrent swath acoustic seafloor surveys as further validation that the substrate dynamics were adequately quantified. Results from both methodologies were in agreement (Storlazzi et al. 2013).

\section{Sampling mobile organisms}

The densities of mobile organisms were quantified using a swath-count protocol developed by PISCO (Hamilton et al. 2010). A diver searched each of 2 transects per plot $(2 \times 30 \mathrm{~m})$ and recorded the identity and abundance of conspicuous mobile species including crustaceans, anthozoans, mollusks, and echino- derms. Sampling was non-destructive and limited to a targeted list of species that were at least $2.5 \mathrm{~cm}$ in greatest dimension.

\section{Analyses of sessile benthic communities}

Seasonal burial and exhumation of rocky reef habitat by sediment can limit or prevent larval settlement, modify competitive interactions, and eliminate sessile organisms. To test if communities of sessile organisms varied in composition among similar rocky reef plots that differ only in their disturbance regime, we analyzed the data using a 3 -factor (i.e. site-fixed, 
plot-fixed, period-fixed) permutational multivariate analysis of variance (PERMANOVA in PRIMER-E; Anderson 2001). Owing to the logistical constraints of site selection to accommodate strict criteria for objective a priori plot selection (adequate size, minimize distance between adjacent plots, similar isobath, and similar sediment dynamics) that restricted the study to 2 sites, we considered 'site' a fixed term. In the absence of logistical restraints, we would recommend selecting random sites and replicating samples from each disturbance regime within sites. Our design was spatially hierarchical, with transects nested within plot (i.e. stable or dynamic) and site (i.e. Lighthouse Point or Terrace Point). We used a classic repeated measures approach to accommodate the repetitive sampling of the same transects, applying a nested design (Quinn \& Keough 2002). Owing to its permutation procedures, PERMANOVA can deal with potential non-independence of samples because the permutation approach effectively ignores correlational structures among samples (Anderson et al. 2008). The number of points occupied by each species in a transect was standardized to the proportion of hard substrate measured by divers, to avoid underestimating the abundance of species obligate to hard substrate. Without this standardization, the reduction of suitable hard substrate by burial would artificially reduce the calculated percent cover of sessile organisms. The standardization also addresses our inability to sample beneath the sand in buried portions of transects. By excluding buried regions from the calculation where we cannot adequately sample sessile organisms, we prevent underestimating the abundance of species that survive beneath sand. Analyses were based on a Bray-Curtis resemblance matrix. We were interested in the results from all crosses, because the responses of sessile communities to sediment burial and exhumation were not expected to be equivalent among sites or periods. To test for heterogeneous dispersion among sites and plots, we performed a PERMDISP analysis in PRIMER. The PERMDISP indicated a significant difference in the dispersions between the 2 sites $(p=0.017$; means: LP $=27.559, \mathrm{TP}=43.396)$; however, the differences in the dispersions differ by less than a factor of 2 and PERMANOVA is not sensitive to heterogeneous dispersion at this level (Anderson \& Walsh 2013). PERMDISP for the plots did not indicate significant differences $(p=0.748$; means: stable $=31.5$, dynamic =28.6).

We used PRIMER v.6 (Clarke \& Gorley 2006) to assess the relative contributions of individual species to differences attributed to the different sediment dynamics. Because we detected an interaction between plots and site in the PERMANOVA using a critical p-value of 0.05 (Anderson 2001) (see 'Results'), we evaluated sites separately. SIMPER was run on UPC counts standardized to rocky reef habitat. We limited the inclusion of species to those contributing cumulatively up to $90 \%$. In this analysis, we were interested in learning the identity of species that show the strongest response to different habitats with respect to burial and exhumation, and to determine whether they were consistent among sites.

\section{Analyses of mobile organisms}

To test for differences in the community structure of benthic mobile organisms, we applied a repeated measures, 3-factor PERMANOVA (as described in the previous section). We square root transformed abundance data to reduce the influence of the most common species (Clarke \& Gorley 2006).

To test for heterogeneous dispersion among sites and plots, we performed a PERMDISP analysis in PRIMER. The PERMDISP indicated significant differences in the dispersion between the 2 sites $(p=0.008$; means: $\mathrm{LP}=24.653$, TP $=34.831$ ). PERMDISP for the plots indicate significant differences in dispersion $(\mathrm{p}=0.035$; means: stable $=26.033$, dynamic $=35.089)$. However, in both cases the differences in the dispersion is less than a factor of 2, and PERMANOVA is not sensitive to heterogeneous dispersion at this level (Anderson \& Walsh 2013).

SIMPER analyses on the transformed data for each site identified the species that contributed to differences between stable and dynamics plots. We restricted the inclusion of species up to a $90 \%$ cumulative contribution cutoff. We used this approach to determine the species that contribute to differences between communities associated with stable versus dynamic plots.

\section{RESULTS}

\section{Substrate burial and exhumation}

In situ measurements of substrate throughout the study demonstrated that our a priori designation of study plots as either stable or dynamic was accurate. Stable plots at both sites persisted as exposed bedrock, whereas dynamic plots exhibited a seasonal pattern of burial (50 to $100 \%$ sediment cover) in summer and full exhumation (0\% sediment cover) in win- 
a)

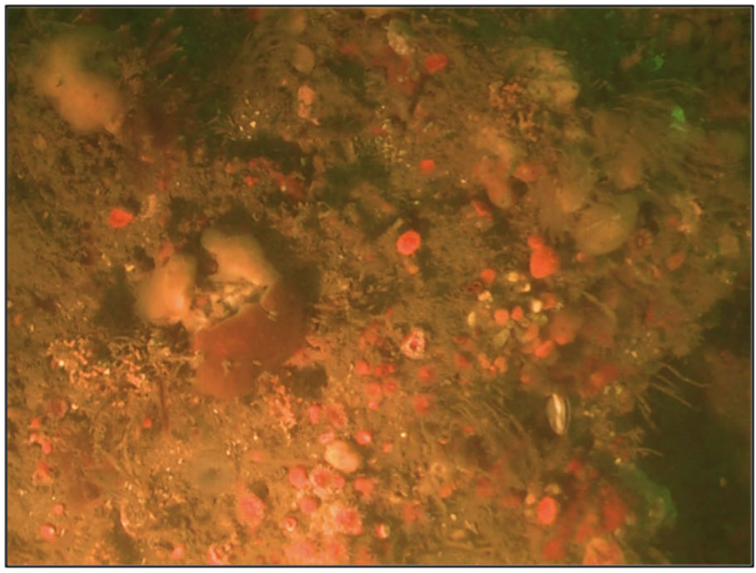

c)

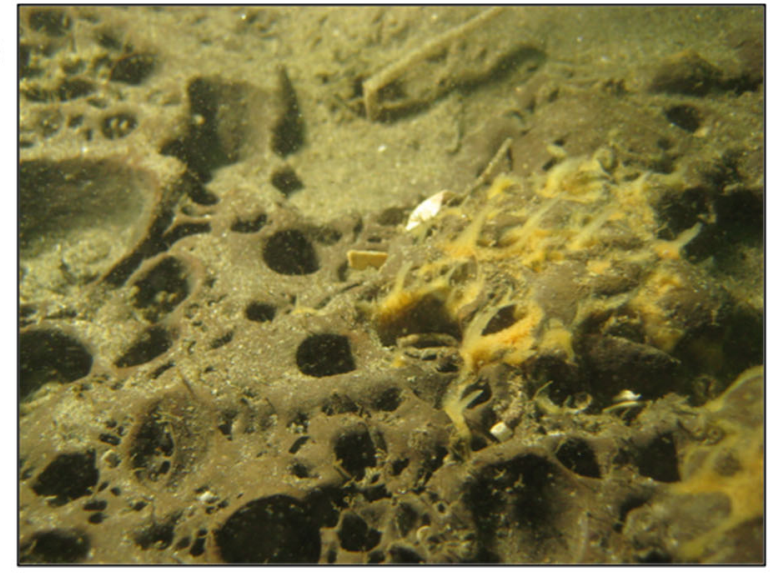

b)

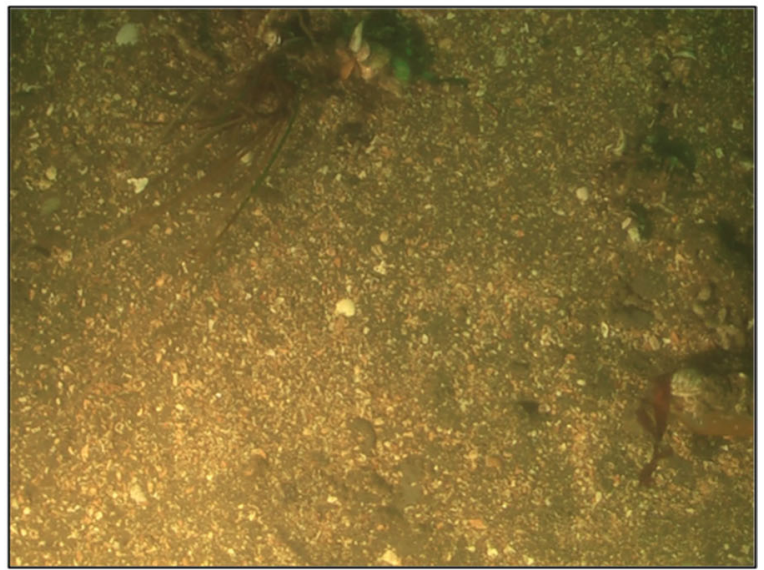

d)

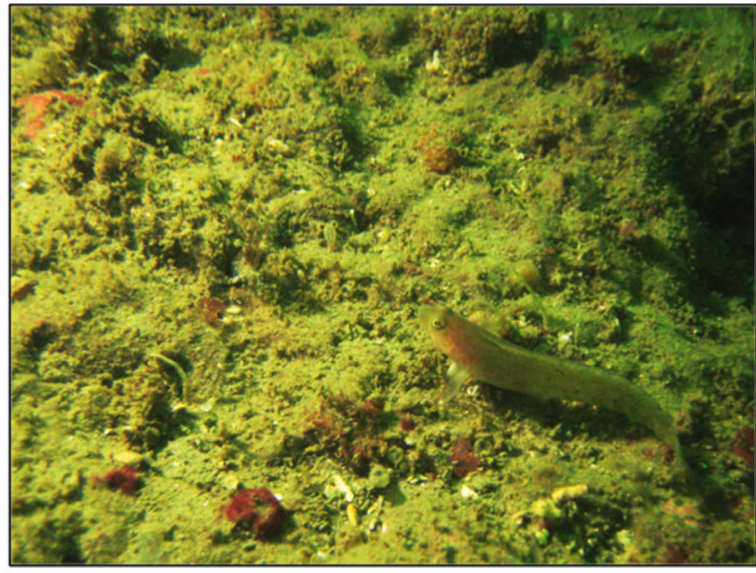

Fig. 2. Example photos of bedrock reef communities taken at the study sites throughout the study. (a) Stable plots are more species rich and populated with sediment-intolerant species such as anemones, cup corals, and filter feeders. Dynamic plots cycle through (b) sand burial and (c,d) exhumation. A recent exhumation of the mudstone reef in (c) demonstrates how extreme disturbance reduces species richness. (d) Communities recover when burial is light and/or infrequent, allowing species to persist or re-establish

Table 1. Results of a 3-factor PERMANOVA testing differences in communities of sessile rock-attached organisms at sites (Lighthouse and Terrace Points), plot (stable and dynamic), and interactions. A repeated measures approach was used to accommodate repetitive sampling of the same transects within plots through time. Counts were standardized by the percentage of rock substrate

\begin{tabular}{|lrrrrr|}
\hline Source & df & SS & \multicolumn{1}{c|}{ MS } & Pseudo- $F$ & p (perm) \\
\hline Between & & & & & \\
Site & 1 & 1980.8 & 1980.8 & 5.97 & 0.032 \\
Plot & 1 & 10470.0 & 10470.0 & 31.55 & 0.031 \\
Plot $\times$ Site & 1 & 1628.8 & 1628.8 & 4.91 & 0.032 \\
Error & 4 & 1327.6 & 331.9 & 0.56 & 0.892 \\
Within & & & & & \\
Period & 2 & 8496.2 & 4248.1 & 7.16 & 0.000 \\
Plot $\times$ Period & 2 & 2821.5 & 1410.7 & 2.38 & 0.052 \\
Site $\times$ Period & 2 & 3084.3 & 1542.2 & 2.60 & 0.037 \\
Plot $\times$ Site $\times$ Period & 2 & 3090.3 & 1545.2 & 2.60 & 0.040 \\
Error & 8 & 4745.4 & 593.2 & & \\
Total & 23 & 37645.0 & & & \\
\hline
\end{tabular}

ter. These results verify that burial and exhumation are sources of physical disturbance for rocky reef communities in this region of Monterey Bay (Fig. 2).

\section{Sessile benthic communities}

A 3-factor repeated measures PERMANOVA analysis for community differences showed that all main effects (Plot, Site, and Period), 2-way interactions (Plot $\times$ Site, Site $\times$ Period), and the 3 -factor interaction were significant (Table 1). Because of the interactions, interpretation of the results is challenging, and therefore it is useful to visualize the results with a non-metric multidimensional scaling plot (Fig. 3). It is clear that despite spatial variation attributed to sites 


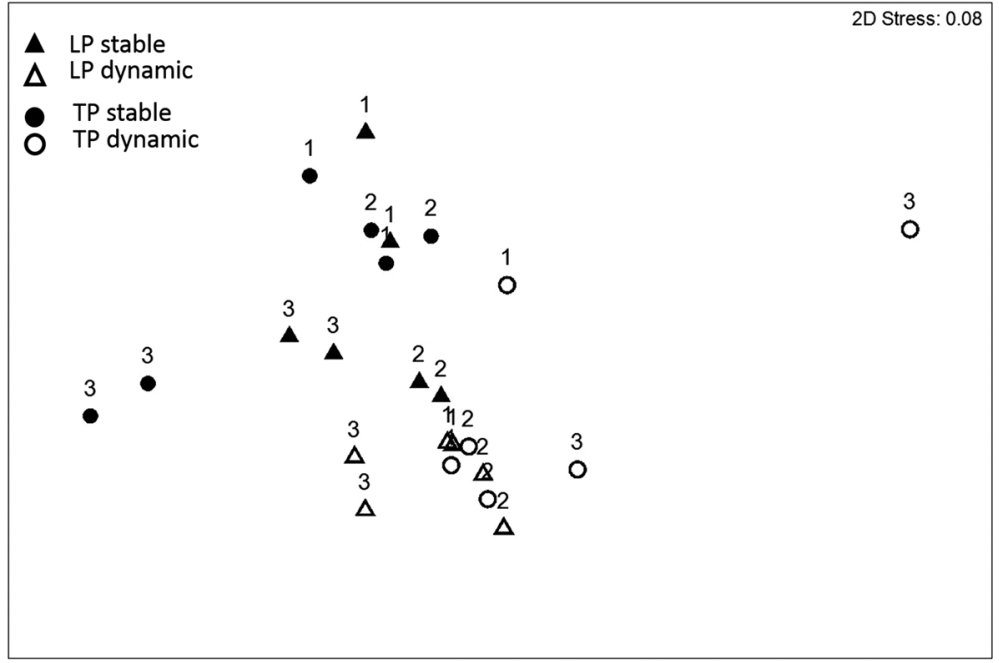

Fig. 3. NMDS plot showing differences in the communities of rockattached sessile organism associations with stable (filled symbols) and dynamic (open symbols) substrates. Individual points are transects. Data were standardized to the amount of bedrock sampled and similarity between transects was determined from a Bray-Curtis resemblance matrix. Data from Terrace Point (TP) and Lighthouse Point (LP) are displayed as circles and triangles, respectively. Sampling periods are indicated by numbers: 1 , winter 2009; 2 , spring 2010; 3, summer 2010

(TP and LP), sessile benthic communities associated with stable rocky reef habitat differ more among stable and dynamic plots than they do among sites. The large dispersion of the data at TP is driven by points from the third sampling period (Fig. 3), suggesting seasonal patterns in community structure at this site that might not have been present at LP. However, significant differences among sampling periods (Table 1) demonstrate that both sites undergo sea-

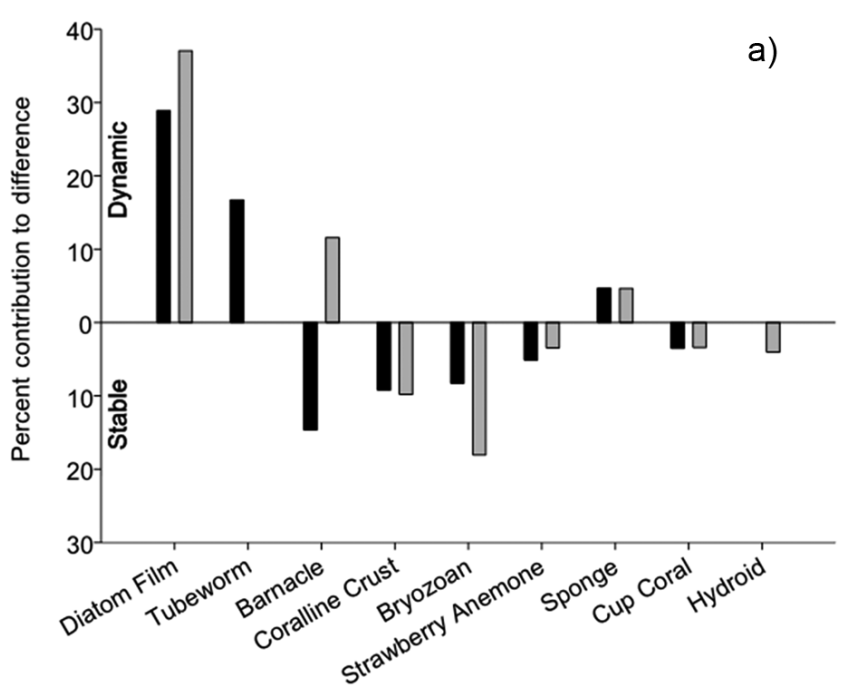

sonal changes in the benthic communities independent of sediment dynamics (e.g. recruitment pulses, community processes). The SIMPER analysis indicated that many of the taxa contributing most to differences between dynamic and stable plots (i.e. diatom films, coralline crust, bryozoans, strawberry anemones, sponges, and cup corals; Fig. 4a) were important at both sites. In contrast, some species were uniquely important at only one of the sites. Tubeworms, for example, contributed $16.7 \%$ to differences among plots at TP, but did not contribute to differences at LP. Similarly, hydroids contributed $4.1 \%$ to differences among plots at LP, but were not important at TP. The direction of the response of the different taxa varied. Diatom films, for example, were more abundant in dynamic plots, whereas bryozoans were more abundant in stable plots. Barnacles explained differences among dynamic and stable plots, but in opposite directions at each site (Fig. 4a).

\section{Mobile benthic communities}

A 3-factor repeated measures PERMANOVA analysis showed that there were significant differences in community structure for the main effects of site and plot (Table 2). Although period was not significant as a main effect, it was significant in 2-way

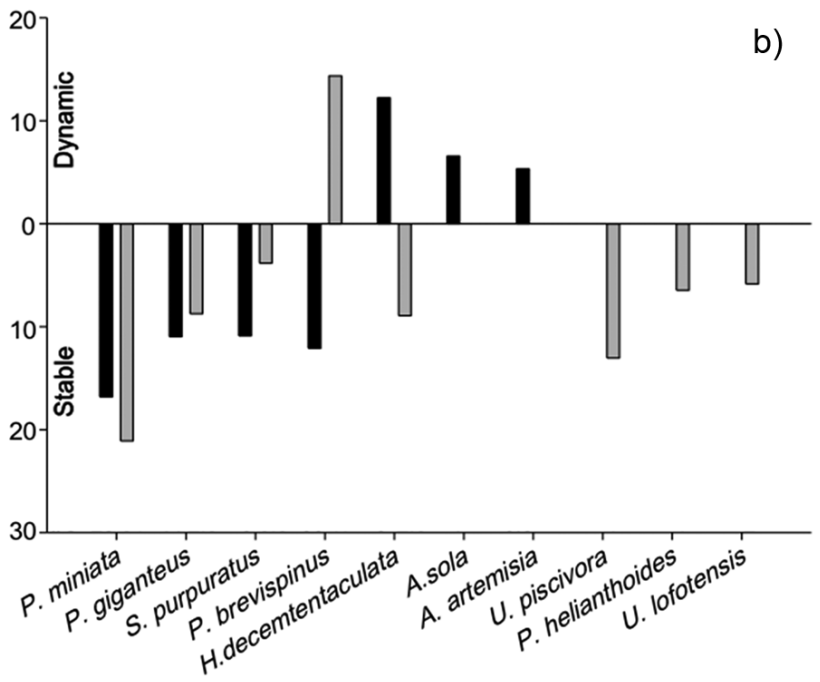

Fig. 4. Contributions of (a) sessile and (b) mobile species (or groups) to differences between communities associated with stable and dynamic plots at Terrace Point (black) and Lighthouse Point (gray). Percent contributions are from SIMPER analyses based on Bray-Curtis similarities 
Table 2. Results of a 3-factor PERMANOVA testing differences in communities of mobile organisms at sites (Lighthouse and Terrace Points), plot (stable and dynamic), and with interactions. A repeated measures approach was used to accommodate repetitive sampling of the same transects within plots through time

\begin{tabular}{|lrrrrr|}
\hline Source & df & SS & MS & Pseudo- $F$ & p (perm) \\
\hline Between & & & & & \\
Site & 1 & 6123.8 & 6123.8 & 12.30 & 0.031 \\
Plot & 1 & 4952.9 & 4952.9 & 9.95 & 0.028 \\
Plot $\times$ Site & 1 & 5668.2 & 5668.2 & 11.39 & 0.031 \\
Error & 4 & 1991.1 & 497.78 & 1.02 & 0.468 \\
Within & & & & & \\
Period & 2 & 1871.3 & 935.64 & 1.92 & 0.103 \\
Plot $\times$ Period & 2 & 1231.5 & 615.74 & 1.26 & 0.304 \\
Site $\times$ Period & 2 & 2663.2 & 1331.6 & 2.73 & 0.021 \\
Plot $\times$ Site $\times$ Period & 2 & 1145.6 & 572.79 & 1.17 & 0.344 \\
Error & 8 & 3906.3 & 488.28 & & \\
Total & 23 & 29554.0 & & & \\
\hline
\end{tabular}

responded oppositely at TP and LP (Fig. 4b). The 3 sea stars $P$. miniata, $P$. brevispinus, and $P$. giganteus were the most abundant species of those that differentiated stable and dynamic plots (Fig. 4b).

\section{DISCUSSION}

Recent advances in high-resolution, swath seafloor acoustic mapping provide scientists with the unprecedented ability to map the distribution of benthic habitats across vast regions of global oceans. These maps have been essential to resource managers for identifying critical fish habitat and for designating Marine Protected Areas (Roff \& Taylor 2000, Harris \& Baker 2012). Most habitat characterizations are based on single surveys; however, our repetitive surveys

interactions with site, indicating that temporal changes of communities varied among sites (Table 2), and specifically that the mobile community at TP was more variable than at LP (Fig. 5). Visualization of the pattern with a non-metric multidimensional scaling plot (NMDS) showed that communities of mobile species differed substantially among stable and dynamic plots (Fig. 5). Communities associated with stable plots were similar between sites, but communities associated with dynamic plots were markedly different (Fig. 5). In addition, LP plots-dynamic and stable - were more closely related to each other than the dynamic and stable plots at TP, as indicated by the greater dispersion of TP data. One-factor SIMPER analyses identified the species contributing to differences in the communities observed in stable and dynamic plots. A total of 10 species contributed up to $90 \%$ of the difference between mobile communities in dynamic and stable plots (Fig. 4b). Half of those species were important at both TP and LP (i.e. Patiria miniata, Pisaster giganteus, Strongylocentrotus purpuratus, Pisaster brevispinus, and Halcampa decemtentaculata), whereas the remaining species (i.e. Anthopleura sola, Urticina piscivora, Pycnopodia helianthoides, and Urticina lofotensis) were discriminating between stable and dynamic plots at only 1 of the 2 sites. For those species important at both sites, $60 \%$ were more abundant in stable plots, and the remaining $40 \%$

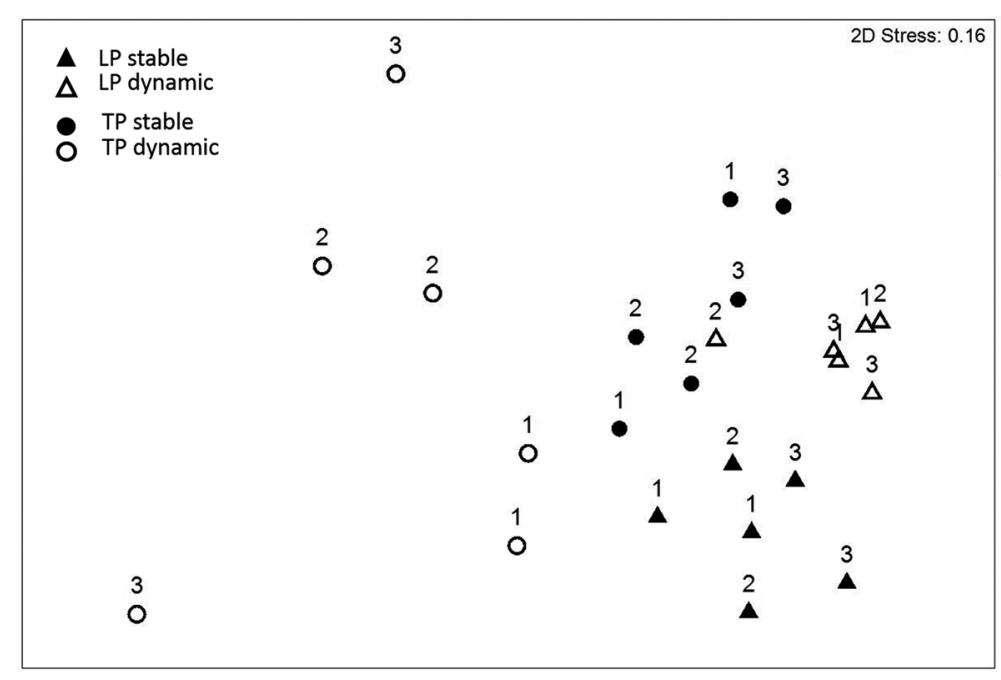

Fig. 5. NMDS plot showing differences in the communities of mobile organisms occurring in stable and dynamic plots. Individual points are transects. Data were square root transformed to reduce the effect of single abundant species and resemblance matrix was based on a Bray-Curtis similarity. 1, winter 2009; 2, spring 2010; 3, summer 2010 
cies diversity and richness of benthic communities was up to $50 \%$ lower in disturbed (i.e. dynamic) versus stable plots (Storlazzi et al. 2013), suggesting that the effects of sediment scour and direct burial are severe for benthic species associated with the rocky reef. We found, however, that a subset of species was favored in dynamic plots that were otherwise rare or absent in stable plots (Fig. 4). These species exhibited a variety of physiological and life history adaptations that contributed to their success in sand-disturbed reef habitats, such as resilient physiology of sessile species (e.g. Polymastia pachymastia), rapid colonization (e.g. diatom films), and mobility (e.g. Pisaster brevispinus). The mosaic of disturbed dynamic and stable substrates, therefore, can increase regional species diversity - a conclusion reinforced by similar studies conducted in the littoral zone (Littler et al. 1983, McQuaid \& Dower 1990).

The negative effects of burial, increased turbidity, and scour associated with seasonal sediment movement can have community-level consequences by influencing all life history stages of benthic species, from settlement to reproduction. Many marine species exhibit a bi-partite life cycle in which larvae disperse broadly, thereby spatially decoupling recruitment from the source of larval production (Carr \& Syms 2006). Community composition at any point in time, therefore, is a function of larval supply, settlement, and survival.

Larvae are often delivered in pulses driven largely by oceanography and both physical and biological cues (Rodriguez et al. 1993). Sediment burial of the rocky reef precludes successful settlement of larvae, whereas subsequent exhumation may favor settlement by exposing bare rock. The timing of burial and exhumation-or the lack of these processes-along with the timing of larval supply is an important contributor to the composition and structure of benthic communities. In this study, for example, a strong pulse of barnacle larvae recruited to both study sites, covering 20 to $60 \%$ of the reef prior to the summer survey. One plot, however, was buried in sediment thereby precluding settlement and/or smothering newly settled barnacles. This single event contributed to strong and persistent differences in the benthic communities between the sites.

After settlement, burial remains a threat to survival for many sessile reef species. Many algae are intolerant of burial because sedimentation inhibits the uptake of nutrients and blocks light required for photosynthesis (Airoldi 2003). Some species of algae, such as coralline algae, can tolerate sediment scour and anoxia from burial, allowing these species to per- sist in dynamic habitats (Eriksson \& Johansson 2005). How coralline algae survive burial is not fully understood, but is possibly associated with a sloughing of epithelial cells once the algae is exhumed (Keats et al. 1997). Despite this remarkable ability, an inverse relationship between sedimentation and the abundance of coralline algae has been documented from hermatypic coral reefs (Fabricius \& De'ath 2001) and reinforced in this study. Although coralline algae occurred at much lower densities in dynamic plots, they were the only algae that survived there. In this case, the ability to survive in stressful environments can impart a strong advantage for tolerant species due to a reduction in competition, particularly where space is limiting.

Suspended sediment and burial can also interfere with metabolic requirements of filter and suspension feeding sessile fauna such as sponges, anemones, corals, hydroids, bryozoans, and ascidians; associated anoxia and fouling of feeding structures can reduce growth rates or increase mortality (Jones et al. 2011). Consistent with this idea, filter and suspension feeders were more abundant in stable plots. Cup corals (e.g. Balanophyllia), anemones (e.g. Corynactis californica), and sponges (e.g. Acanthancora cyanocrypta) were typically more abundant in stable plots and found on rises above the seafloor where the effects of scour and burial were reduced. Within taxa, however, there were exceptions. A single species of sponge, $P$. pachymastia, seems tolerant of both sediment scour and burial, and was more abundant in dynamic plots. $P$. pachymastia can survive episodic burial due to raised oscula that extend through and beyond the sediment layer, allowing the sponge to filter feed and respire while most of the animal is buried. Cnidarians, such as cup corals and anemones, live attached to the rocky reef, but some cnidarians typically live partially buried in sediment, such as the infaunal anemone Halcampa decemtentaculata. These examples highlight the difficulty of generalizing across taxa and emphasize the need to understand how individual species are influenced by scour and burial, especially when trying to predict benthic community structure from seafloor maps generated from remote sensing data.

In contrast to sessile species, individuals of mobile species can seek out favorable habitats and avoid unfavorable ones. This allows for a post-settlement redistribution of these species and contributes to more variable community structure over short timescales compared to sessile communities. Many of the mobile species in this study were more abundant in stable plots, a distribution likely driven by the abun- 
dance of their primary prey on stable rocky reef habitat. Some species were more common in disturbed habitats, often when burial was shallow or the reef was recently exposed. Species such as $P$. brevispinus opportunistically aggregate at the sediment-reef interface to scavenge sessile organisms killed by burial and scour.

The dynamics of burial and scour in coastal oceans has been described for decades, but only recently have tools become available to quantify and map seafloor dynamics over meaningful spatial and temporal scales. This research represents one of the first attempts to understand how burial and exhumation influence the structure of bedrock reef communities over a seasonal timescale. Sediment dynamics are important for structuring benthic communities. Disturbance changed local species composition and favored a subset of sediment-tolerant reef species and early colonizers. The spatial mosaic of substrate dynamics therefore may bolster regional diversity; however, more research is needed to fully understand how benthic reef communities respond across the range of disturbance conditions (e.g. burial frequency, intensity, duration, and associated processes such as turbidity, scour, and sedimentation).

Predicting benthic communities from seafloor maps promises to be an important tool for resource managers (e.g. identifying essential fish habitat, stock assessments, and designation of Marine Protected Areas). In its application, however, we caution that changing seafloor landscapes and their influence on benthic communities should be considered. Constraints on the design of this study restricted its scope of inference, but we believe it is a good case study that is representative of processes that operate ubiquitously in coastal oceans. We encourage further field studies to inform and validate map-based predictions, and to understand how burial, scour, and turbidity shape the structure and function of nearshore benthic communities at local and regional scales.

Acknowledgements. The work was funded by the US Geological Survey Coastal and Marine Geology Program's Benthic Habitats (Pacific) Project to better understand the effects of geologic and oceanographic processes on coastal and marine ecosystems, and by the Partnership for Interdisciplinary Studies of the Coastal Oceans (PISCO). We thank Mark Carr (UCSC), Pete Raimondi (UCSC), and Melissa Foley (USGS) for their comments and advice that greatly improved the quality of this manuscript. This is contribution number 466 from PISCO, funded primarily by the David and Lucile Packard Foundation and the Gordon and Betty Moore Foundation. Use of trademark names does not imply USGS endorsement of products.

\section{LITERATURE CITED}

Airoldi L (2003) The effects of sedimentation on rocky coast assemblages. Oceanogr Mar Biol Annu Rev 41:161-236

Anderson MJ (2001) A new method for non-parametric multivariate analysis of variance. Austral Ecol 26:32-46

> Anderson MJ, Walsh DC (2013) PERMANOVA, ANOSIM, and the Mantel test in the face of heterogeneous dispersions: what null hypothesis are you testing? Ecol Monogr 83:557-574

Anderson MJ, Gorley RN, Clarke KR (2008) PERMANOVA + for PRIMER: guide to software and statistical methods. PRIMER-E, Plymouth

Anima RJ, Eittreim SL, Edwards BD, Stevenson AJ (2002) Nearshore morphology and Late Quaternary geologic framework of the northern Monterey Bay Marine Sanctuary, California. Mar Geol 181:35-54

Carr MH, Syms C (2006) Recruitment. In: Allen LG, Pondella DJ II, Horn MH (eds) The ecology of marine fishes: California and adjacent waters. University of California Press, Berkeley, CA, p 411-427

Clarke KR, Gorley RN (2006) PRIMER v.6: user manual/ tutorial. PRIMER-E, Plymouth

Connell JH (1978) Diversity in tropical rain forests and coral reefs. Science 199:1302-1310

Denny MW (1987) Life in the maelstrom: the biomechanics of wave-swept rocky shores. Trends Ecol Evol 2:61-66

> Denny M, Gaylord B (2002) The mechanics of wave-swept algae. J Exp Biol 205:1355-1362

$>$ Eriksson BK, Johansson G (2005) Effects of sedimentation on macroalgae: species-specific responses are related to reproductive traits. Oecologia 143:438-448

> Fabricius K, De'ath G (2001) Environmental factors associated with the spatial distribution of crustose coralline algae on the Great Barrier Reef. Coral Reefs 19:303-309

Field ME (2005) Living with change: response of the sea floor to natural events. In: Barnes PW, Thomas JP (eds) Benthic habitats and the effects of fishing. Proc Am Fish Soc Symp 41. American Fisheries Society, Bethesda, MD, p 215-218

Foster MS, Schiel DR (1985) The ecology of giant kelp forests in California: a community profile. Biological Report 85 (7.2). US Fish and Wildlife Service, Slidell, LA

$>$ Fox JW (2013) The intermediate disturbance hypothesis should be abandoned. Trends Ecol Evol 28:86-92

Hall SJ (1994) Physical disturbance and marine benthic communities: life in unconsolidated sediments. Oceanogr Mar Biol Annu Rev 32:179-239

> Hamilton SL, Caselle JE, Malone DP, Carr MH (2010) Incorporating biogeography into evaluations of the Channel Islands marine reserve network. Proc Natl Acad Sci USA 107:18272-18277

- Harris PT (2014) Shelf and deep-sea sedimentary environments and physical benthic disturbance regimes: a review and synthesis. Mar Geol 353:169-184

Harris PT, Baker EK (2012) GeoHab atlas of seafloor geomorphic features and benthic habitats: synthesis and lessons learned. In: Harris PT, Baker EK (eds) Seafloor geomorphology as benthic habitat. Elsevier, London, p 871-890

> Harris PT, Hughes MG (2012) Predicted benthic disturbance regimes on the Australian continental shelf: a modelling approach. Mar Ecol Prog Ser 449:13-25

> Jones HFE, Pilditch CA, Bruesewitz DA, Lohrer AM (2011) Sedimentary environment influences the effect of an infaunal suspension feeding bivalve on estuar- 
ine ecosystem function. PLoS ONE 6:e27065

Keats DW, Knight MA, Pueschel CM (1997) Antifouling effects of epithallial shedding in three crustose coralline algae (Rhodophyta, Coralinales) on a coral reef. J Exp Mar Biol Ecol 213:281-293

Littler MM, Martz DR, Littler DS (1983) Effects of recurrent sand deposition on rocky intertidal organisms: importance of substrate heterogeneity in a fluctuating environment. Mar Ecol Prog Ser 11:129-139

McQuaid CD, Dower KM (1990) Enhancement of habitat heterogeneity and species richness on rocky shores inundated by sand. Oecologia 84:142-144

Menge BA, Sutherland JP (1987) Community regulation: variation in disturbance, competition, and predation in relation to environmental stress and recruitment. Am Nat 130:730-757

Quinn G, Keough M (2002) Experimental design and data analysis for biologists. Cambridge University Press, Cambridge

Rodriguez SR, Ojeda FP, Inestrosa NC (1993) Settlement of benthic marine invertebrates. Mar Ecol Prog Ser 97: 193-207

Roff JC, Taylor ME (2000) National frameworks for marine conservation-a hierarchical geophysical approach. Aquat Conserv 10:209-223

Roxburgh SH, Shea K, Wilson JB (2004) The intermediate disturbance hypothesis: patch dynamics and mechanisms of species coexistence. Ecology 85:359-371

Ryan DA, Brooke BP, Collins LB, Kendrick GA and others (2007) The influence of geomorphology and sedimentary processes on shallow-water benthic habitat distribution: Esperance Bay, Western Australia. Estuar Coast Shelf Sci 72:379-386

Schiel DR, Foster MS (2015) The biology and ecology of giant kelp forests. University of California Press, Oakland, CA

Editorial responsibility: Lisandro Benedetti-Cecchi, Pisa, Italy
Sousa WP (1984) The role of disturbance in natural communities. Annu Rev Ecol Syst 15:353-391

Sousa WP (2001) Natural disturbance and the dynamics of marine benthic communities. In: Bertness MD, Gaines SD, Hay ME (eds) Marine community ecology. Sinauer Associates, Sunderland, MA, 85-130

Storlazzi CD, Jaffe BE (2002) Flow and sediment suspension events on the inner shelf of central California. Mar Geol 181:195-213

Storlazzi CD, Fregoso TA, Golden NE, Finlayson DP (2011) Sediment dynamics and the burial and exhumation of bedrock reefs along an emergent coastline as elucidated by repetitive sonar surveys: northern Monterey Bay, CA. Mar Geol 289:46-59

Storlazzi CD, Fregoso TA, Figurski JD, Freiwald J, Lonhart SI, Finlayson DP (2013) Burial and exhumation of temperate bedrock reefs as elucidated by repetitive highresolution sea floor sonar surveys: spatial patterns and impacts to species' richness and diversity. Cont Shelf Res 55:40-51

Svensson JR, Lindegarth M, Pavia H (2009) Equal rates of disturbance cause different patterns of diversity. Ecology 90:496-505

Thistle D (1981) Natural physical disturbances and communities of marine soft bottoms. Mar Ecol Prog Ser 6: 223-228

Thistle D (2003) The deep-sea floor: an overview. In: Tyler PA (ed) Ecosystems of the world, Book 28. Elsevier Science, Amsterdam, p 5-37

> Wingfield DK, Storlazzi CD (2007) Spatial and temporal variability in oceanographic and meteorologic forcing along central California and its implications on nearshore processes. J Mar Syst 68:457-472

Wu J, Loucks OL (1995) From balance of nature to hierarchical patch dynamics: a paradigm shift in ecology. Q Rev Biol 70:439-466

Submitted: October 13, 2014; Accepted: May 10, 2016 Proofs received from author(s): June 16, 2016 